\title{
Parsimonious Sidelobe Control for Transmit Beamforming Using Multidimensional Arrays
}

\author{
Aboulnasr Hassanien*, Moeness G. Amin, Yimin D. Zhang, and Fauzia Ahmad \\ Center for Advanced Communications, Villanova University, Villanova, PA 19085, USA
}

\begin{abstract}
Two-dimensional (2D) transmit beamforming aims at focusing the transmitted energy within ceratin desired sector while minimizing the amount of energy in the out-of-sector regions. In this paper, we propose parsimonious formulations to the sidelobe control problem in 2D transmit beamforming with multidimensional arrays. The out-of-sector region is partitioned into a small number of subsectors where the subspace spanned by the steering vectors associated with the spatial directions within a certain subsecetor is approximated by the effective discreteprolate spheroidal sequences associated with that subsector. Then, the sidelobe control is achieved by imposing constraints on the magnitude of the inner product between the 2D transmit beamforming weight vector and the discrete-prolate spheroidal sequences. Simulations examples are presented which show the effectiveness of the proposed formulations.
\end{abstract}

Keywords: Two-dimensional transmit beamforming, sidelobe control, convex optimization

\section{INTRODUCTION}

In the last decade, multiple-input multiple-output (MIMO) radar has been the focus of intensive research [1]-[8]. The advantages of MIMO radar with colocated antennas include improved angular resolution and parameter identifiability, increased number of detectable targets, and extended array aperture by virtual sensors. However, without employing proper transmit beamforming, MIMO radar suffers from the loss of transmit processing gain. To address the latter problem, the concepts of phased-MIMO radar and transmit energy focussing have been introduced [5], [7], [9]-[15]. However, the aforementioned methods are primarily developed for the case of MIMO radar with one-dimensional transmit array. In practice, multidimensional arrays are often used to enable parameter estimation and target localization in the two-dimensional (2D) azimuth-elevation domain. The number of transmit elements used in a 2D array is typically large and can be in the range of several thousands. Motivated by practical considerations, several methods for $2 \mathrm{D}$ transmit beamforming design using multidimensional arrays have been reported in the literature [16]-[19] which adopt a convex optimization based approach. Sidelobe control is achieved via enforcing sidelobe constraints at a $2 \mathrm{D}$ discrete grid. The use of a coarse grid results in power radiation leakage towards directions located between the coarse grid points. On the other hand, the use of a fine grid to approximate the sidelobe region results in a very high number of constraints which requires a high computational cost.

In this paper, we propose parsimonious formulations to the problem of $2 \mathrm{D}$ transmit beamforming design and sidelobe control using multidimensional arrays. We adopt the concept of focusing the transmit energy within the desired sector while enforcing constraints on the amount of energy leakage in the out-of-sector area. To achieve good sidelobe control without having to enforce sidelobe constraints over a fine grid, we divide the out-of-sector regions into a small number of subsectors. The subspace spanned by the steering vectors associated with the spatial directions within a certain subregion is approximated by a small number of discrete-prolate spheroidal sequences (DPSS). Then, the sidelobe control is achieved by imposing constraints on the magnitude of the inner product between the $2 \mathrm{D}$ transmit beamforming weight vector and the DPSSs. The resulting problem is formulated as a convex optimization problem with a small number of sidelobe constraints enabling a computationally efficient solution. We use simulation examples to validate the effectiveness of the proposed parsimonious $2 \mathrm{D}$ transmit beamforming design method.

This paper is organized as follows. In Section 2, we present the signal model and formulate the 2D transmit beamforming design problem. In Section 3, we present the proposed parsimonious 2D transmit beamforming design method. Simulation results are provided in Section 4, while conclusions are drawn in Section 5.

*aboulnasr.hassanien@villanova.edu 


\section{PROBLEM FORMULATION}

We Consider a MIMO radar system equipped with $M_{T}$ transmit antennas arranged in an arbitrary shape in a three-dimensional space. In a Cartesian coordinate system, the $m$ th transmit antenna is assumed to be located at the position $\mathbf{d}_{m}=\left[x_{m}, y_{m}, z_{m}\right]^{T}, m=1, \ldots, M_{T}$, where $(\cdot)^{T}$ stands for the transpose. The $M_{T} \times 1$ steering vector of the transmit array is defined as

$$
\mathbf{a}(\theta, \phi)=\left[e^{-j 2 \pi \mathbf{q}^{H} \mathbf{d}_{1}}, \ldots, e^{-j 2 \pi \mathbf{q}^{H} \mathbf{d}_{M_{T}}}\right]^{T},
$$

where $\theta$ and $\phi$ denote the elevation and azimuth angles, respectively, $\mathbf{q} \triangleq[\sin \theta \cos \phi, \sin \theta \sin \phi, \cos \theta]$, and $(\cdot)^{H}$ stands for the Hermitian. Without loss of generality, we consider the case of a transmit array being an $M \times N$ antenna uniform rectangular array (URA), where $M$ is the number of antenna elements in a given row and $N$ is the number of antenna elements in a given column. The elements on any given column in the transmit array are assumed to be equally spaced with interelement spacing $d_{x}$ while the interelement spacing between any two adjacent elements on any row is given by $d_{y}$. The $M N \times 1$ steering vector of the transmit array is represented as $[17]$

$$
\mathbf{a}(\eta, \zeta)=\operatorname{vec}\left(\mathbf{Z} \odot\left[\mathbf{u}(\eta) \mathbf{v}^{T}(\zeta)\right]\right)
$$

where $\zeta \triangleq \sin \theta \sin \phi$ and $\eta \triangleq \sin \theta \cos \phi$, respectively, $\mathbf{Z}$ is an $M \times N$ matrix of ones and zeros where the $m n$-th entry equals zero if the $m n$-th element of the array is absent, $\operatorname{vec}(\cdot)$ stands for the operator that stacks the columns of a matrix in one column vector, $\odot$ stands for the Hadamard product, and $\mathbf{u}$ and $\mathbf{v}$ are vectors of dimension $M \times 1$ and $N \times 1$, respectively, which are defined as follows

$$
\begin{aligned}
& \mathbf{u}(\eta)=\left[1, e^{-j 2 \pi d_{x} \eta}, \ldots, e^{-j 2 \pi\left(M_{t}-1\right) d_{x} \eta}\right]^{T} \\
& \mathbf{v}(\zeta)=\left[1, e^{-j 2 \pi d_{y} \zeta}, \ldots, e^{-j 2 \pi\left(N_{t}-1\right) d_{y} \zeta}\right]^{T}
\end{aligned}
$$

Let $\mathbf{w}$ be the $M N \times 12 \mathrm{D}$ transmit beamforing weight vector. The $2 \mathrm{D}$ transmit power radiation pattern is defined as

$$
G(\eta, \zeta)=\left|\mathbf{w}^{H} \mathbf{a}(\eta, \zeta)\right|^{2}
$$

The 2D transmit beamforming design problem can be stated as follows. Given the structure of the transmit array, design the transmit beamforming weight vector $\mathbf{w}$ which fits a certain desired 2D transmit radiation pattern $G_{d}(\eta, \zeta)$ at an affordable computational burden.

\section{PARSIMONIOUS 2D TRANSMIT BEAMFORMING DESIGN}

In this section, we present the 2D transmit beamforming design formulations and the associated parsimonious implementation. One meaningful way to design the transmit beamforming weight vector w is to minimize the difference between the actual 2D transmit power radiation pattern and the desired one while keeping the highest sidelobe level below a certain predetermined level. This can be formulation as the following constrained optimization problem

$$
\begin{aligned}
& \left.\min _{\mathbf{w}} \max _{\eta, \zeta}|| G_{d}(\eta, \zeta)|-| \mathbf{w}^{H} \mathbf{a}(\eta, \zeta)\right|^{2} \mid, \quad \eta \in \Pi, \zeta \in \Omega \\
& \text { subject to }\left|\mathbf{w}^{H} \mathbf{a}(\eta, \zeta)\right|^{2} \leq \Delta, \quad \eta \in \Pi_{s}, \quad \zeta \in \Omega_{s},
\end{aligned}
$$

where $\Pi=\left[\eta_{\min }, \eta_{\max }\right]$ and $\Omega=\left[\zeta_{\min }, \zeta_{\max }\right]$ define the desired sector, $\Pi_{s}$ and $\Omega_{s}$ define the out-of-sector regions, respectively, and $\Delta$ is a positive number of user choice used to control the sidelobe level. The optimization problem (6)-(7) is non-convex and, therefore, cannot be solved in a computationally efficient manner. A semidefinite 
relaxation technique [20], [21] can be used to recast it as a convex one. Introducing the new variable $\mathbf{X}=\mathbf{w} \mathbf{w}^{H}$, the optimization problem (6)-(7) can be reformulated as [17]

$$
\begin{aligned}
\min _{\mathbf{X}} \max _{\eta_{i}, \zeta_{j}} & || G_{d}\left(\eta_{i}, \zeta_{j}\right)\left|-\operatorname{trace}\left\{\mathbf{X a}\left(\eta_{i}, \zeta_{j}\right) \mathbf{a}^{H}\left(\eta_{i}, \zeta_{j}\right)\right\}\right|, \quad \eta_{i} \in \Pi, i=1, \ldots, I, \zeta_{j} \in \Omega, j=1, \ldots, J \\
\text { subject to } & \left|\mathbf{w}^{H} \mathbf{a}\left(\eta_{q}, \zeta_{p}\right)\right|^{2} \leq \Delta, \quad \eta_{q} \in \Pi_{s}, q=1, \ldots, Q, \zeta_{p} \in \Omega_{s}, p=1, \ldots, P, \\
& \mathbf{X} \succeq 0,
\end{aligned}
$$

where $\eta_{i}, i=1, \ldots, I, \zeta_{j}, j=1, \ldots, J, \eta_{q}, q=1, \ldots, Q$, and $\zeta_{p}, p=1, \ldots, P$ are continuum grids chosen uniformly (or non-uniformly) to approximate the sectors $\Pi, \Omega, \Pi_{s}$, and $\Omega_{s}$, respectively. The optimization problem (8)-(10) is convex and can be solved in a computationally efficient manner using the interior point methods [22].

An alternative way to solve (6)-(7) in a computationally efficient manner is based on defining the desired $2 \mathrm{D}$ transmit radiation pattern within the sector-of-interest in terms of both magnitude and phase, that is, $G_{d}(\eta, \zeta)=\left|G_{d}(\eta, \zeta)\right| e^{-j 2 \pi\left(\gamma_{1} \eta+\gamma_{2} \zeta\right)}, \eta \in \Pi, \zeta \in \Omega$, where $\gamma_{1}$ and $\gamma_{2}$ are constants used to control the rate of change of the desired phase profile of $G_{d}(\eta, \zeta)$. Therefore, the non-convex optimization problem (6)-(7) can be reformulated in the following convex form

$$
\begin{aligned}
& \min _{\mathbf{w}} \max _{\eta_{i}, \zeta_{j}}|| G_{d}\left(\eta_{i}, \zeta_{j}\right)\left|e^{-j 2 \pi\left(\gamma_{1} \eta_{i}+\gamma_{2} \zeta_{j}\right)}-\mathbf{w}^{H} \mathbf{a}\left(\eta_{i}, \zeta_{j}\right)\right|^{2}, \quad \eta_{i} \in \Pi, i=1, \ldots, I, \zeta_{j} \in \Omega, j=1, \ldots, J \\
& \text { subject to }\left|\mathbf{w}^{H} \mathbf{a}\left(\eta_{q}, \zeta_{p}\right)\right|^{2} \leq \Delta, \quad \eta_{q} \in \Pi_{s}, q=1, \ldots, Q, \zeta_{p} \in \Omega_{s}, p=1, \ldots, P .
\end{aligned}
$$

In is worth noting that the decision variable $\mathbf{X}$ in (8)-(10) is of dimension $M N \times M N$ while the decision variable $\mathbf{w}$ in (11)-(12) is of dimensionality $M N \times 1$. Therefore, the computational burden associated with solving the latter optimization problem is relatively cheaper than the computational burden associated with solving the former optimization problem.

\subsection{Parsimonious Sidelobe Control}

The sidelobe regions, i.e., $\Pi_{s}$ and $\Omega_{s}$, usually occupy more space than the desired sector regions $\Pi$ and $\Omega$. Therefore, enforcing sidelobe control over a fine grid, i.e., choosing $Q$ and $P$ to be large numbers of directions, results in a large number of constraints which, in turn, increases the computational load associated with solving the optimization problem. On the other hand, enforcing sidelobe control over a coarse grid, i.e., choosing $Q$ and $P$ to be small numbers, does not enable sufficient sidelobe control in between the grid points. To solve this problem, we propose the use of DPSS for sidelobe control.

Let us partition the out-of-sector regions $\Pi_{s}$ and $\Omega_{s}$ into $K(K \ll Q)$ and $L(L \ll P)$ subsectors, respectively. For each subsector $\Pi_{k}$ and $\Omega_{l}$, the following non-negative matrices can be built

$$
\mathbf{A}_{k, l}=\int_{\Pi_{k}} \int_{\Omega_{l}} \mathbf{a}(\eta, \zeta) \mathbf{a}^{H}(\eta, \zeta) d \eta d \zeta, \quad k=1, \ldots, K, \quad l=1, \ldots, L .
$$

A good subspace representation for the $(k, l)$ th subsector is given by the significant spheroidal sequences associated with that subsector. This subspace can be constructed as

$$
\mathbf{U}_{k, l}=\left[\mathbf{e}_{1}, \ldots, \mathbf{e}_{E}\right],
$$

where $\left\{\mathbf{e}_{i}\right\}_{i=1}^{E}$ are $E$ principal eigenvectors of $\mathbf{A}_{k, l}$. Note that the number of effective eigenvectors can be taken as the number of effective eigenvalues of the matrix $\mathbf{A}_{k, l}$, i.e., the $E$ largest eigenvalues whose sum exceeds a certain percentage (e.g., $99 \%$ ) of the total sum of all eigenvalues.

A parsimonious $2 \mathrm{D}$ transmit beamforming design can be achieved by reformulating (11)-(12) as the following optimization problem

$$
\begin{aligned}
& \min _{\mathbf{w}} \max _{\eta_{i}, \zeta_{j}}|| G_{d}\left(\eta_{i}, \zeta_{j}\right)\left|e^{-j 2 \pi\left(\gamma_{1} \eta_{i}+\gamma_{2} \zeta_{j}\right)}-\mathbf{w}^{H} \mathbf{a}\left(\eta_{i}, \zeta_{j}\right)\right|^{2}, \quad \eta_{i} \in \Pi, i=1, \ldots, I, \zeta_{j} \in \Omega, j=1, \ldots, J \\
& \text { subject to }\left\|\mathbf{w}^{H} \mathbf{U}_{k, l}\right\| \leq \Delta_{\text {DPSS }}, \quad k=1, \ldots, K, \quad l=1, \ldots, L,
\end{aligned}
$$


where $\Delta_{\text {DPSS }}$ is a positive number used to control the sidelobe level. By choosing $K \ll Q$ and $L \ll P$ to be small, the number of constraints involved in (15)-(16) is small and, therefore, the associated computational cost is reduced as compared to that of solving (11)-(12).

\section{SIMULATION RESULTS}

In our simulations, we assume a uniform rectangular transmit array of size $7 \times 7$. The desired spatial sector is defined as $\theta \in\left[0^{\circ}, 11.5^{\circ}\right]$ and $\phi \in\left[0^{\circ}, 360^{\circ}\right]$, i.e., the sector represents a cone of width $23^{\circ}$ centered around the

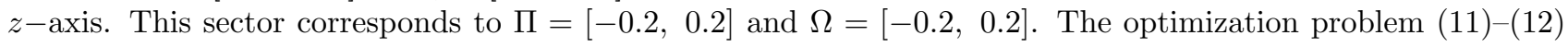
is solved using a coarse grid of $3 \times 3$ for each subsector, i.e., the whole out-of-sector region is approximated by $8 \times 3 \times 3=72$ grid points. The same problem is solved using a fine grid of $21 \times 21$ for each subsector, i.e., the whole out-of-sector region is approximated by a fine grid of $8 \times 21 \times 21=3528$ points. For the parsimonious formulations, the out-of-sector region is partitioned into 72 non-overlapping subsectors. Each subsector is chosen to be centered around one of the 72 coarse grid points. Numerical integration is used to calculate 72 non-negative matrices; one for each subsector. The parsimonious based solution is obtained by solving (15)-(16). $E=10$ effective eigenvalues are assumed. $\Delta=0.1$ is assumed throughout this paper. Figs. 1 and 2 show the transmit radiation patterns obtained by solving (11)-(12) using the coarse and the fine grids, respectively. It is clear from the figures that the use of a coarse grid does not enable controlling the sidelobe level between the grid points. Fig. 3 shows the transmit radiation pattern obtained using the parsimonious formulations (15)-(16). The figure shows that the parsimonious based solution achieves a transmit radiation pattern that is as good as that obtained using the case of fine gird but with complexity comparable to that required for the case of a coarse grid.

The same simulation example is repeated using an array of size $15 \times 15$ transmit elements. Note that, in this case, the transmit weight vector $\mathbf{w}$ will be of dimensionality $225 \times 1$ which means the degrees of freedom is increased as compared to the previous example. Figs. 4 and 5 show the transmit radiations patterns obtained

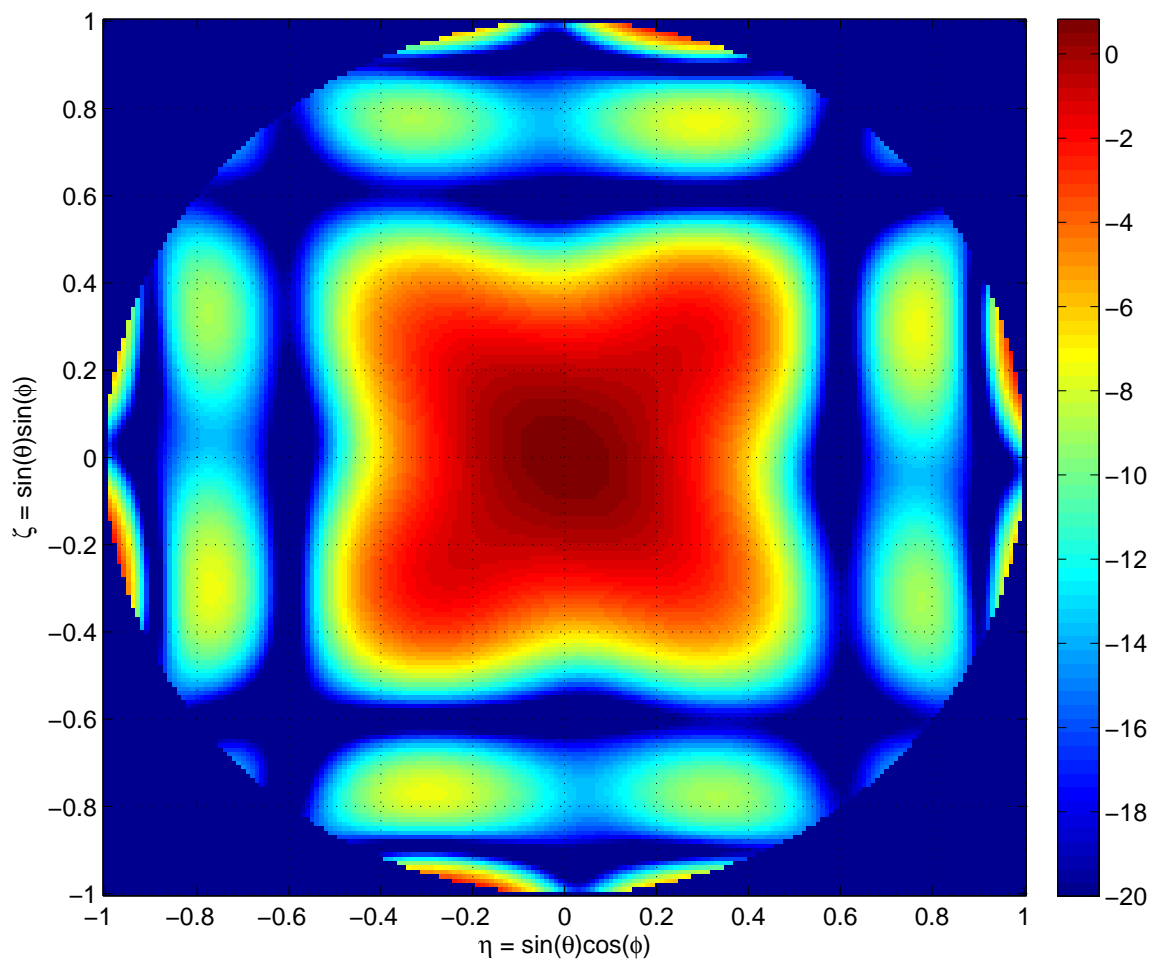

Figure 1. $2 \mathrm{D}$ transmit power radiation pattern in $\mathrm{dB}, 7 \times 7 \mathrm{URA}$, coarse grid. 


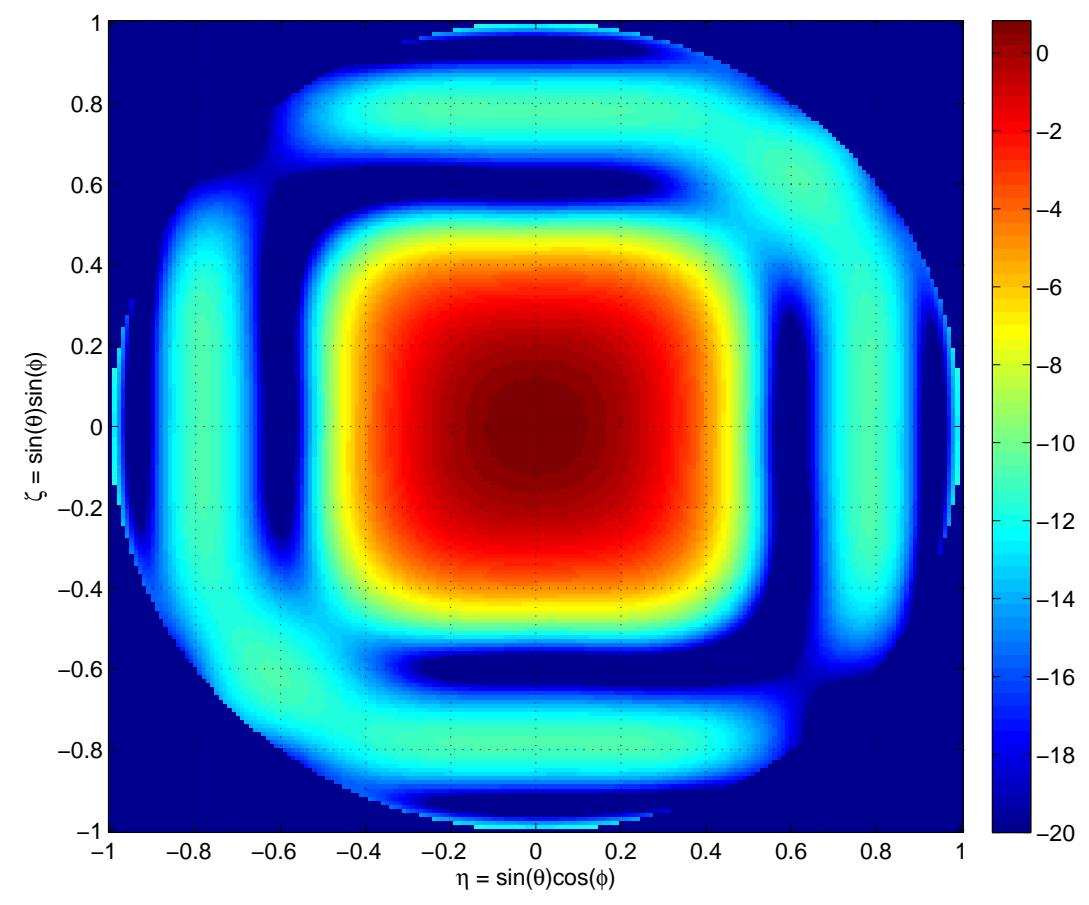

Figure 2. $2 \mathrm{D}$ transmit power radiation pattern in $\mathrm{dB}, 7 \times 7 \mathrm{URA}$, fine grid.

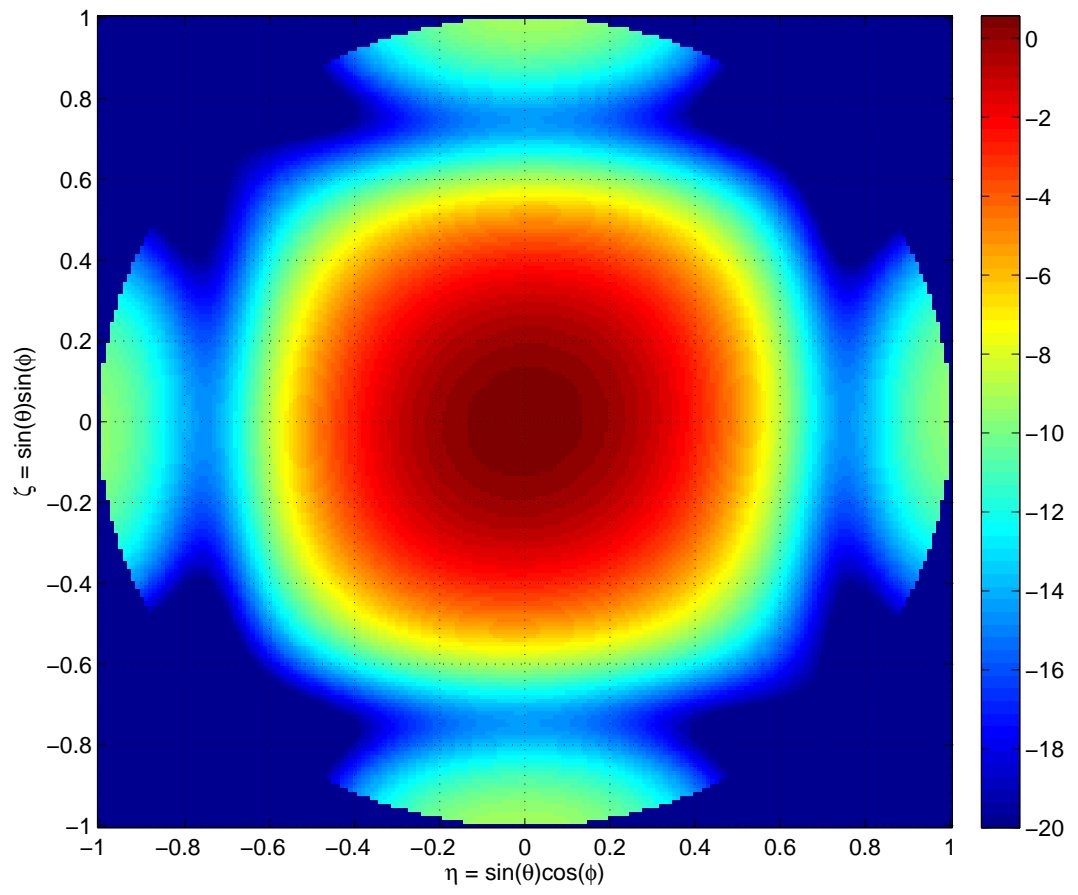

Figure 3. 2D transmit power radiation pattern in $\mathrm{dB}, 7 \times 7 \mathrm{URA}$, parsimonious based design. 


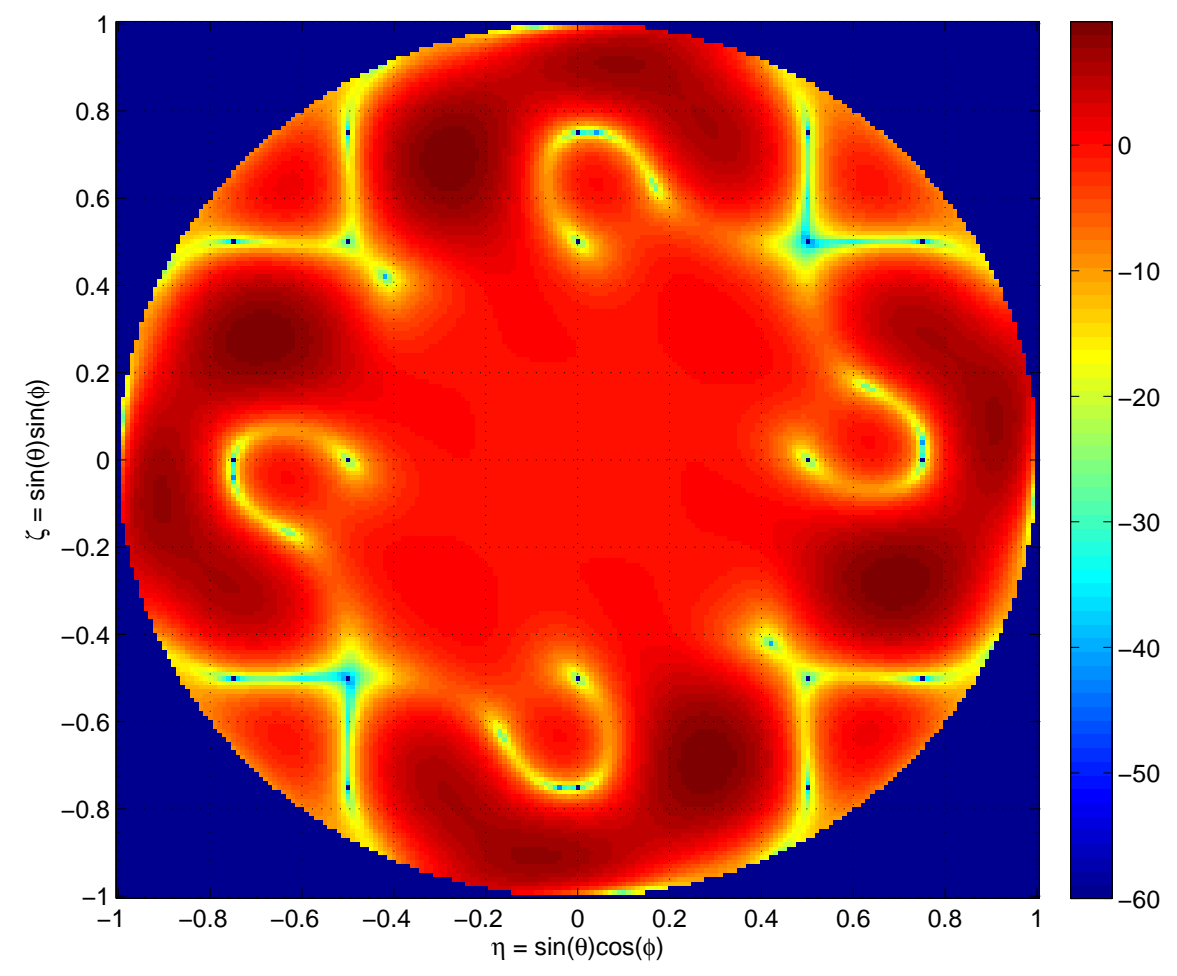

Figure 4. 2D transmit power radiation pattern in $\mathrm{dB}, 15 \times 15 \mathrm{URA}$, coarse grid.

using the same coarse and fine grids used in the previous example. The increased number of degrees of freedom enables the optimization solver to better enforce the constraints while solving (11)-(12) at the price of having higher sidelobes between the grid points. This explains why the solution obtained using a coarse grid yields sidelobe levels between the grid points that are higher than the main lobe of the patten as shown in Fig. 4. On the other hand, Fig. 5 shows that the use of a fine grid enables achieving good sidelobe control at the price of higher computational cost. Finally, Fig. 6 shows the transmit radiation pattern for the parsimonious based design obtained by solving (15)-(16). The figure illustrates that the parsimonious based solution is as good as the solution obtained using a fine grid. In is worth noting that in practice very large arrays are used and, therefore, the computational cost associated with the use of fine grid is prohibitive. Therefore, for very large arrays, the proposed parsimonious based 2D transmit beamforming design represents a computationally attractive choice.

\section{CONCLUSIONS}

The problem of two-dimensional (2D) transmit beamforming using multidimensional arrays was considered. The concept of focusing the transmitted energy within ceratin desired sector while minimizing the amount of energy wasted in the out-of-sector regions was adopted. Parsimonious formulations to the sidelobe control problem in 2D transmit beamforming were proposed. The out-of-sector region is partitioned into a small number of subsectors where the subspace spanned by the steering vectors associated with the spatial directions within a certain subsecetor is approximated by the effective discrete-prolate spheroidal sequences associated with that subsector. Sidelobe control was achieved by imposing constraints on the magnitude of the inner product between the 2D transmit beamforming weight vector and the discrete-prolate spheroidal sequences. The proposed parsimonious formulations as shown to achieve a transmit radiation pattern that is as good as the on obtained using a fine grid. The effectiveness of the proposed formulations was shown using simulations examples. 


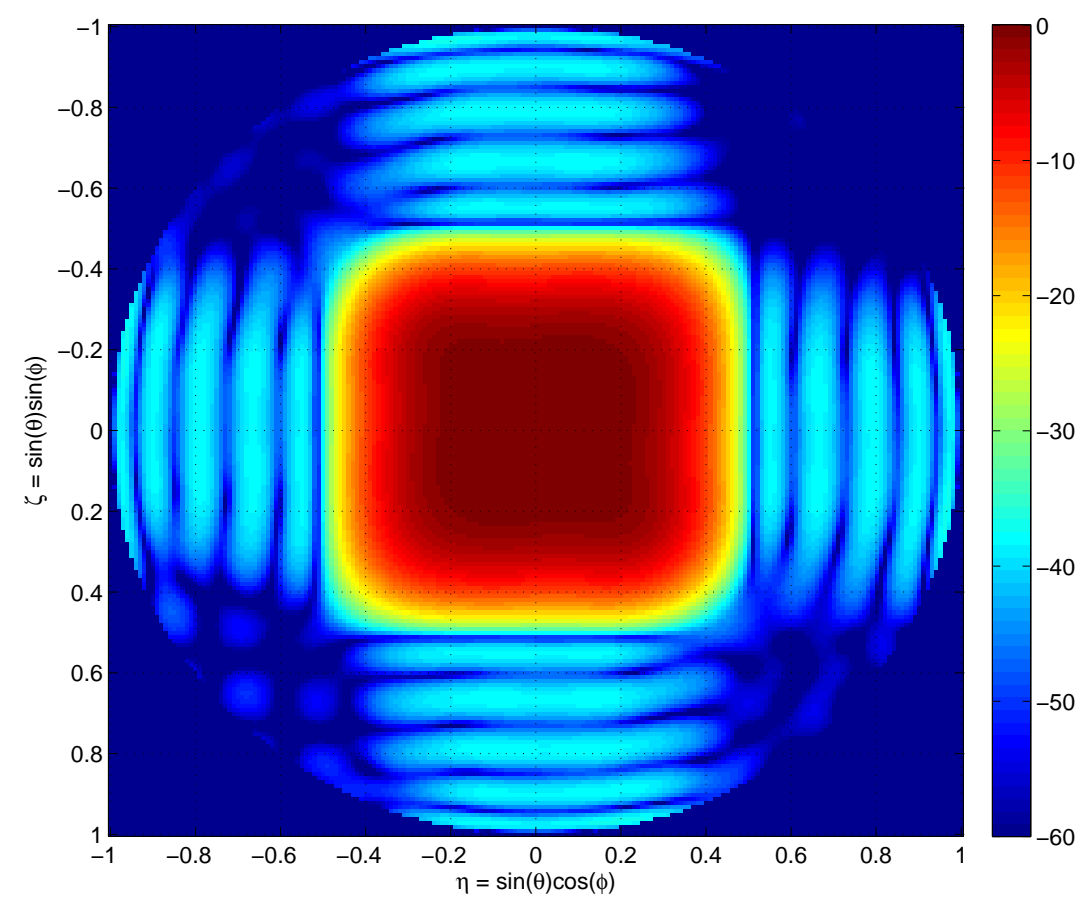

Figure 5. 2D transmit power radiation pattern in $\mathrm{dB}, 15 \times 15 \mathrm{URA}$, fine grid.

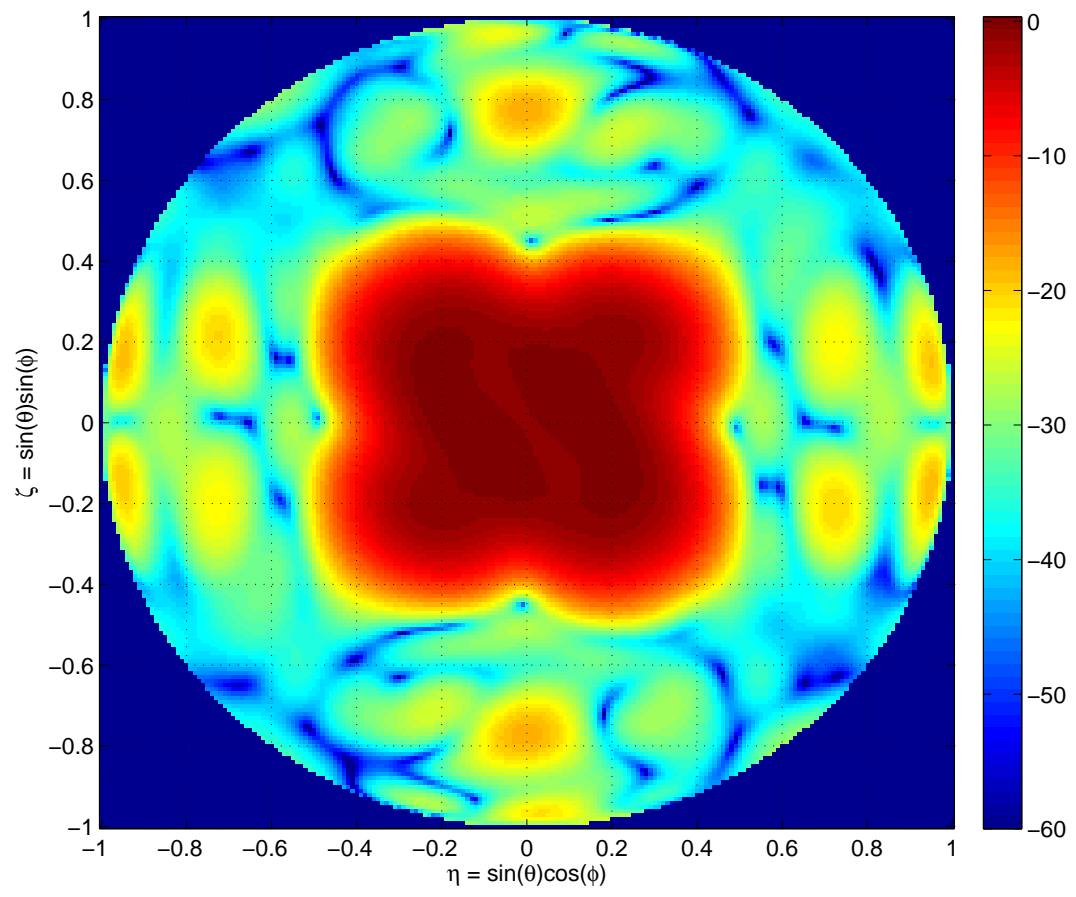

Figure 6. $2 \mathrm{D}$ transmit power radiation pattern in $\mathrm{dB}, 15 \times 15 \mathrm{URA}$, parsimonious based design. 


\section{REFERENCES}

[1] E. Fishler, A. Haimovich, R. Blum, D. Chizhik, L. Cimini, and R. Valenzuela, "MIMO radar: An idea whose time has come," in Proc. IEEE Radar, Honolulu, HI, Apr. 2004, pp. 71-78.

[2] I. Bekkerman and J. Tabrikian, "Target detection and localization using MIMO radars and sonars," IEEE Trans. Signal Process., vol. 54, no. 10 pp. 3873-3883, Oct. 2006.

[3] J. Li and P. Stoica, "MIMO radar with colocated antennas," IEEE Signal Process. Mag., vol. 24, pp. 106114, Sep. 2007.

[4] J. Li and P. Stoica, MIMO Radar Signal Processing. New Jersy: Wiley, 2009.

[5] A. Hassanien and S. Vorobyov, "Phased-MIMO radar: A tradeoff between phased-array and MIMO radars," IEEE Trans. Signal Process., vol. 58, no. 6, pp. 3137-3151, Jun. 2010.

[6] A. Hassanien and S. A. Vorobyov, "Why the phased-MIMO radar outperforms the phased-array and MIMO radars," in Proc. European Signal Process. Conf., Aalborg, Denmark, Aug. 2010, pp. 1234-1238.

[7] A. Hassanien and S. Vorobyov, "Transmit energy focusing for DOA estimation in MIMO radar with colocated antennas," IEEE Trans. Signal Process., vol. 59, no. 6, pp. 2669-2682, June 2011.

[8] Y. D. Zhang, M. G. Amin, and B. Himed, "Joint DOD/DOA estimation in MIMO radar exploiting timefrequency signal representations," EURASIP Journal on Advances in Signal Process., pp. 1-10, July 2012.

[9] P. Stoica, J. Li, and Y. Xie, "On probing signal design for MIMO radar," IEEE Trans. Signal Processin, vol. 55, no. 8, pp. 4151-4161, Aug. 2007.

[10] D. Fuhrmann and G. San Antonio, "Transmit beamforming for MIMO radar systems using signal crosscorrelation," IEEE Trans. Aerospace and Electronic Systems, vol. 44, no. 1, pp. 1-16, Jan. 2008.

[11] A. Hassanien and S. Vorobyov, "Transmit/receive beamforming for MIMO radar with colocated antennas," in Proc. IEEE Int. Conf. Acoust., Speech, Signal Process., Taipei, Taiwan, Apr. 2009, pp. 2089-2092.

[12] T. Aittomaki and V. Koivunen, "Beampattern optimization by minimization of quartic polynomial," in Proc. IEEE/SP Statist. Signal Processing Workshop, Cardiff, U.K., Sep. 2009, pp. 437-440.

[13] A. Hassanien and S. Vorobyov, "Subspace-based direction finding using transmit energy focusing in MIMO radar with colocated antennas," in Proc. IEEE Int. Conf. Acoust., Speech, Signal Process., Prague, Czech Republic, May 2011, pp. 2788-2791.

[14] A. Khabbazibasmenj, A. Hassanien, and S. Vorobyov, "Transmit beamspace design for direction finding in colocated MIMO radar with arbitrary receive array," in Proc. IEEE Int. Conf. Acoust., Speech, Signal Process., Prague, Czech Republic, May 2011, pp. 2784-2787.

[15] A. Khabbazibasmenj, A. Hassanien, S. Vorobyov, and M. Morency, "Efficient transmit beamspace design for search-free based DOA estimation in MIMO radar," IEEE Trans. Signal Process., vol. 62, no. 3, pp. 14901500, Mar. 2014.

[16] A. Hassanien, S. A. Vorobyov, Y-S. Yoon, and J-Y. Park, "Root-MUSIC based source localization using transmit array interpolation in MIMO radar with arbitrary planar arrays," in Proc. IEEE Int. Workshop on Computational Advances in Multi-Sensor Adaptive Process., Saint Martin, Dec. 2013

[17] A. Hassanien, M. W. Morency, A. Khabbazibasmenj, S. A. Vorobyov, J.-Y. Park, and S.-J. Kim, "Twodimensional transmit beamforming for MIMO radar with sparse symmetric arrays," in Proc. IEEE Radar Conf., Ottawa, ON, Canada, Apr.-May 2013.

[18] A. Hassanien, S. A. Vorobyov, and J.-Y. Park, "Joint transmit array interpolation and transmit beamforming for source localization in MIMO radar with arbitrary arrays," in Proc. IEEE Int. Conf. Acoust., Speech, Signal Process., Vancouver, BC, Canada, May 2013, pp. 4139-4143.

[19] A. Hassanien, S. A. Vorobyov, S.-J. Kim, and J.-Y. Park, "Two-stage based design for phased-MIMO radar with improved coherent transmit processing gain," in Proc. IEEE Int. Workshop Signal Process. Advances in Wireless Comm., Toronto, ON, Canada, June 2014, pp. 45-49.

[20] Z.-Q. Luo, W.-K. Ma, A. M.-C. So, Y. Ye, and S. Zhang, "Semidefinite relaxation of quadratic optimization problems," IEEE Signal Process. Magaz., vol. 27, no. 3, pp. 20-34, May 2010.

[21] K. T. Phan, S. A. Vorobyov, N. D. Sidiropoulos, and C. Tellambura, "Spectrum sharing in wireless networks via QoS-aware secondary multicast beamforming," IEEE Trans. Signal Process., vol. 57, no. 6, pp. 23232335, June 2009.

[22] S. Boyd and L. Vandenberghe, Convex Optimization. Cambridge University Press, 2009. 